\title{
Relationship between body composition and eating disorder risk in adolescents. UP\& DOWN follow-up substudy
}

\author{
J.A. Rodriguez-Laprast $^{1}$, S. Gomez-Martinez ${ }^{1}$, A. Hernandez ${ }^{1}$, V. Cabanas-Sanchez ${ }^{2}$, \\ O.L. Veiga ${ }^{2}$ and A. Marcos ${ }^{1}$ \\ ${ }^{1}$ Institute of Food Science, Technology and Nutrition. Spanish National Research Council, Madrid, Spain and \\ ${ }^{2}$ Department of Physical Education, Sport and Human Movement. Autonomous University of Madrid, Madrid, Spain
}

\section{Abstract}

Adolescence is a decisive and characteristic period for overall development throughout the life-span in which Eating Disorders (ED) are among the most common mental health problems in young population. Different factors might be related to the risk of ED such as sex and body composition, but literature about hydration status is still very scarce. This study was aimed to assess possible relationships between ED risk and body composition, including hydration status. A 2-year follow-up substudy from the UP\&DOWN Study was performed in a sample of 77 adolescents at baseline ( $\mathrm{n}=74$ after the follow-up; $13.87 \pm 1.54$ years old at baseline). Subjects were grouped by sex. ED risk was measured by the SCOFF questionnaire, a validated screening tool for ED. Risk of ED criterion is considered when SCOFF score is equal or higher than 2.0. Body composition, particularly Fat Mass (FM\%) and hydration [Total Body Water (TBW in liters) regarding the weight of individuals and its range (low, within, high)] were determined by bioimpedance. Data analysis was conducted using version 25.0; SPSS Inc., Chicago, IL, USA, software. A logistic regression was made to select the variables [sex, TBW (L), FM\%] that could be more related to ED risk, considered as a dependent variable. Crosstabs and chi-square test were used to establish the relationships between the categorical variables. Almost $30 \%$ girls and $10 \%$ boys were at risk of ED at baseline $(\mathrm{P}=0.036)$. Furthermore, almost $39 \%$ girls and none of the boys were at ED risk after the follow-up $(\mathrm{P}=0.000)$. All the subjects at $\mathrm{ED}$ risk showed a low TBW range at both points (baseline, $\mathrm{P}=0.04$; after the follow-up, $\mathrm{P}=0.039$ ). A probit binary model of repeated measures (151 healthy adolescent values were obtained considering every subject in both points of the study) was made to study the influence of sex, TBW (L), FM\% on the ED risk. Positive associations between Sex-TBW interaction and ED risk (P $=0.000)$ as well as between $F M \%$ and ED risk $(P=0.020)$ were observed. These findings show that body composition, not only fat mass but also hydration status is related to ED risk, although it is well known that boys and girls show different hydration and fat patterns. The fact of being a female adolescent with low TBW and FM\% values may predispose to a high ED risk. Therefore, sex-hydration interaction together with body composition should be further investigated for ED in adolescence.

\section{Conflict of Interest}

"There is no conflict of interest". 